\title{
Structures of electromagnetic type on vector bundles
}

\author{
E. Reyes, V. Cruceanu \& P.M. Gadea
}

\begin{abstract}
Structures of electromagnetic type on a vector bundle are introduced and studied. The metric case is also defined and studied. The sets of compatible connections are determined and a canonical connection is defined.
\end{abstract}

\section{Introduction}

Structures of electromagnetic type (em-structures) and structures of metric electromagnetic type (mem-structures) on a manifold were progressively introduced in $[9,11,7]$ (see also $[6]$ ) and studied in detail in $[5,7,8,13,14]$. In the present paper we define similar structures for the case of a vector bundle $\xi=(E, \pi, M)$, and relate them to product, complex, para-Hermitian, Hermitian, para-Kähler or indefinite Kähler, structures. (In the sequel, by a pseudo-Riemannian metric we shall understand a metric of any signature, and by an indefinite (metric) structure a structure including a pseudo-Riemannian metric.) Then, we determine the set of connections on $\xi$ compatible with those structures and we introduce a canonical connection. Considering an almost para-Hermitian (resp. indefinite Hermitian) structure on the base manifold $M$ and an indefinite Hermitian (resp. para-Hermitian) structure of the bundle $\xi$, we prove that the corresponding diagonal lift of these structures, with respect to a connection on $\xi$, are mem-structures on the total space $E$. Finally, some properties of those mem-structures are established.

We recall the physical origin of the topic $([9,11])$. Let $M^{4}$ be a spacetime of general relativity, with gravitational tensor $g$ of signature -+++ . Let $F$ be the electromagnetic field of type $(0,2)$, which is skewsymmetric, that is a 2 -form. Setting $F(X, Y)=g(J X, Y)$, the tensor field $J$ so defined is the electromagnetic tensor field of type $(1,1)$ associated to $F$. We have $g(J X, Y)+g(X, J Y)=0$. The characteristic equation of $J$ is $\operatorname{det}(J-\lambda I)=0$, which is satisfied by $J$, and we have

$$
J^{4}+2 k J^{2}+l I=0, \quad k=-\frac{1}{4} \operatorname{trace} J^{2}, \quad l=\operatorname{det} J .
$$

If $x \in M^{4}$, it is said that $J_{x}$ is of $1^{s t}, 2^{n d}$, or $3^{r d}$ class at $x$ if, respectively,

$$
l_{x} \neq 0, \quad l_{x}=0, k_{x} \neq 0, \quad l_{x}=0, k_{x}=0 .
$$


It is said that $J$ is of $1^{\text {st }}, 2^{\text {nd }}$, or $3^{\text {rd }}$ class if it is of such class at every $x$. The characteristic polynomial of the second class is $J^{2}\left(J^{2}+2 k\right)$, but the minimal polynomial is $J\left(J^{2}+2 k\right)$, so that the condition $J\left(J^{2}+2 k\right)=0$ characterizes the second class. The field of an electromagnetic plane wave is of $3^{r d}$ class. The field of a moving electron is of $2^{\text {nd }}$ class. More complicated fields belong to the $1^{\text {st }}$ class. The equation one gets from the minimal polynomial in the $1^{\text {st }}$ class is

$$
\left(J^{2}-f^{2}\right)\left(J^{2}+h^{2}\right)=0 .
$$

with $f, h$ nowhere-vanishing $C^{\infty}$ functions on $M^{4}$. Such a tensor field $J$ on a general manifold $M$ determines a $G$-structure on $M$.

To handle the nonconstant local cross-section situation corresponding to (1.1), one can use the relationships among $G$-structures, related sections of an associated bundle and functions of certain kind on $M$, as follows: Let $\left(\mathcal{P}, \pi_{P}, M, H\right)$ be a principal bundle with group $H, H \times W \rightarrow W$ a left action of $H$ on a manifold $W$, and $\left(E=\mathcal{P} \times_{H} W, \pi_{E}, M, W\right)$ the associated bundle. A $J$-subset $S$ of $W$ with corresponding group $G$, a subgroup of $H$, is defined by the conditions: (1) $S \subset$ fixpoint set of $G,(2) h \in H, h(S) \cap S \neq \emptyset \Rightarrow h \in G$. For instance, points are $J$-subsets with $G$ the corresponding isotropy group. A cross-section $K$ of $\pi_{E}$ is a $J$-section if it can be locally represented as the "product" of a cross-section $\sigma$ of $\pi_{P}$ and a $S$-valued function $\widetilde{K}$, so that

$$
K_{x}=\sigma_{x} \cdot \widetilde{K}_{x}=\text { equivalence class of }\left(\sigma_{x}, \widetilde{K}_{x}\right) \text { in } E .
$$

Then $\widetilde{K}$ is globally defined, and the $\sigma$ generate a principal subbundle of $\mathcal{P}$. $K$ is a constant $J$-section if and only if $\widetilde{K}$ is constant. Different sections can generate the same subbundle, and in fact, every principal subbundle can be generated by a constant $J$-section.

Now, let $\mathcal{P}$ be the principal bundle of frames over $M$, so that $H=G L(n, \mathbb{R})$, and let $W$ be a real vector space. If $J \in W$ is given with the conditions stated above, a $J$-section generates a $J$-structure with group $G$, which is a $G$-structure. The tensor $K$ has in principle variable components in adapted frames. This is a slight generalization with respect to the usually considered $G$-structures, given by tensors with constant components, which here correspond to constant $J$-sections. Since every $J$-structure is generated by some constant $J$-section, this generalization is useless for the study of the $J$-structure itself; but if the emphasis shifts to the study of variable $J$-sections, the results are significant, specially with respect to the parallelizability of the tensors.

In the particular case of a $(1,1)$ tensor field $J$ satisfying $\left(J^{2}-f^{2}\right)\left(J^{2}+h^{2}\right)=$ 0 , with characteristic polynomial $(x-p)^{r_{1}}(x-p)^{r_{2}}\left(x^{2}+q^{2}\right)^{s}, r_{1}, r_{2}, s \geq 1$, $r_{1}+r_{2}+2 s=n=\operatorname{dim} M$, the $J$-subset consists of matrices of the form

$$
\left(\begin{array}{cccc}
p I_{r_{1}} & & & \\
& -p I_{r_{2}} & & \\
& & & -q I_{s} \\
& & q I_{s} &
\end{array}\right)
$$


and the structural group is $G=G L\left(r_{1}, \mathbb{R}\right) \times G L\left(r_{2}, \mathbb{R}\right) \times G L(s, \mathbb{C})$. It is proved ([7]) that the $G$-structure defined by $J$ above is also defined by a tensor field, say again $J$, satisfying $\left(J^{2}-1\right)\left(J^{2}+1\right)=0$, that is, the relation $J^{4}=1$ considered in the present paper.

Notice that the G-structure is exactly the same, not an associated or equivalent one. In the 4-dimensional case the group reduces to $G=G L(1, \mathbb{R}) \times$ $G L(1, \mathbb{R}) \times G L(1, \mathbb{C})$. It is also proved $([7])$ that there exists an adapted Riemannian metric so that the group can be reduced to $G=O\left(r_{1}\right) \times O\left(r_{2}\right) \times U(s)$, and in the 4 -dimensional case to $\mathbb{Z}_{2} \times \mathbb{Z}_{2} \times U(1)$, that is, essentially to the unitary group $U(1)$.

\section{Structures of electromagnetic type on a vector bundle}

Let $\xi=(E, \pi, M)$ be a $C^{\infty}$ vector bundle with total space $E$ and projection map $\pi$ over a connected paracompact base manifold $M$. The rank of $E$ is the (common) dimension of the fibres. Let $C^{\infty}(M)$ denote the ring of real functions, $\mathcal{T}_{q}^{p}(M)$ the $C^{\infty}(M)$-module of $(p, q)$-tensor fields, and $\mathcal{T}(M)$ the $C^{\infty}(M)$-tensor algebra of $M$. We respectively denote by $\mathcal{T}_{q}^{p}(\xi)$ and $\mathcal{T}(\xi)$ the $C^{\infty}(M)$-module of tensor fields of type $(p, q)$ and the $C^{\infty}(M)$-tensor algebra of the bundle $\xi$.

We recall that an almost product (resp. almost complex) structure on a manifold $M$ is defined by a tensor field $J$ of type $(1,1)$ satisfying $J^{2}=I$ (resp. $J^{2}=-I$ ). An almost para-Hermitian (resp. indefinite almost Hermitian) structure on $M$ is defined by a couple $(J, g)$, given by an almost product (resp. almost complex) structure $J$ and a pseudo-Riemannian metric compatible with $J$ in the sense that $g(J X, Y)+g(X, J Y)=0, X, Y \in \mathfrak{X}(M)$; that is, as an anti-isometry (resp. isometry). A para-Kähler (resp. indefinite Kähler) manifold is a manifold $M$ endowed with an almost para-Hermitian (resp. indefinite almost Hermitian) structure such that the Levi-Civita connection of $g$ parallelizes $J$.

Definition 2.1. A structure of electromagnetic type on $\xi=(E, \pi, M)$ is an $M$-endomorphism $J$ of $\xi$ satisfying

$$
J^{4}=I,
$$

with characteristic polynomial $(x-1)^{r_{1}}(x+1)^{r_{2}}\left(x^{2}+1\right)^{s}$, where $r_{1}, r_{2}, s$ are constants greater than or equal to 1 such that $r_{1}+r_{2}+2 s=\operatorname{rank} E$.

Setting $P=J^{2}$, we have $P^{2}=I$, so $P$ is a product structure on $\xi$, admitting $J$ as a "square root". Conversely, if $P$ is a product structure admitting a "square root" $J$, then $J$ is an em-structure on $\xi$. Denoting by $\xi_{1}$ and $\xi_{2}$ respectively the +1 and -1 eigen-subbundles of $P$, it is easy to see that $\xi_{1}$ and $\xi_{2}$ are invariant by $J$ and that $J_{1}=\left.J\right|_{\xi_{1}}$ defines a product structure of $\xi_{1}$ and $J_{2}=\left.J\right|_{\xi_{2}}$ a complex structure of $\xi_{2}$. So, one has

$$
\xi=\xi_{1} \oplus \xi_{2}, \quad J=J_{1} \oplus J_{2} .
$$


Conversely, if $\xi_{1}$ and $\xi_{2}$ are two supplementary subbundles of $\xi, J_{1}$ is a product structure of $\xi_{1}$, and $J_{2}$ a complex structure of $\xi_{2}$, then $J=J_{1} \oplus J_{2}$ is an em-structure on $\xi$. Denoting by $P_{1}$ and $P_{2}$ the projections of $\xi$ on $\xi_{1}$ and $\xi_{2}$ respectively, we obtain

$$
P=P_{1}-P_{2}, \quad J=J_{1} \circ P_{1}+J_{2} \circ P_{2} .
$$

Summing up we have

Proposition 2.1. An em-structure on the vector bundle $\xi=(E, \pi, M)$ can be defined by each one of the following conditions:

(1) An $M$-endomorphism $J$ of $\xi$ satisfying $J^{4}=I$,

(2) A product structure $P$ of $\xi$ admitting a "square root" J,

(3) Two supplementary subbundles $\xi_{1}$ and $\xi_{2}$ of $\xi$ respectively endowed with a product structure and a complex structure.

Remark 2.1. A product structure $P$ which admits a "square root" is a particular one because $\operatorname{rank} \xi_{2}$ must be even.

Definition 2.2. A structure of metric electromagnetic type (mem-structure) on the vector bundle $\xi$ is a pair $(J, g)$, where $J$ is an em-structure and $g$ a pseudo-Riemannian metric on $\xi$ satisfying the compability condition

$$
g(J X, Y)+g(X, J Y)=0, \quad X, Y \in \xi .
$$

Denoting by $\delta_{J}$ the derivation defined by $J$ in the tensor algebra $\mathcal{T}(\xi)$, the relation $(2.2)$ can be written as

$$
\delta_{J} g=0,
$$

from which it follows $g(P X, P Y)=g(X, Y), X, Y \in \mathfrak{X}(M)$. Therefore, the pair $(P, g)$ is a pseudo-Riemannian product structure of $\xi$ and so the subbundles $\xi_{1}$ and $\xi_{2}$ are mutually orthogonal with respect to $g$. Denoting respectively by $g_{1}$ and $g_{2}$ the restrictions of $g$ to $\xi_{1}$ and $\xi_{2}$, from (2.2) we obtain

$$
\delta_{J_{1}} g_{1}=0, \quad \delta_{J_{2}} g_{2}=0,
$$

which may be written

$$
g_{1}\left(J_{1} X, J_{1} X\right)=-g_{1}(X, Y), \quad g_{2}\left(J_{2} X, J_{2} Y\right)=g_{2}(X, Y), \quad X, Y \in \mathfrak{X}(\xi) .
$$

Hence $\left(J_{1}, g_{1}\right)$ is a para-Hermitian structure of $\xi_{1}$ and $\left(J_{2}, g_{2}\right)$ is an indefinite Hermitian structure of $\xi_{2}$. Conversely, if $\xi_{1}$ and $\xi_{2}$ are two supplementary subbundles of $\xi$ such that $\xi_{1}$ is endowed with a para-Hermitian structure $\left(J_{1}, g_{1}\right)$ and $\xi_{2}$ with an indefinite Hermitian structure $\left(J_{2}, g_{2}\right)$, then considering $J$ as given by (2.1) and setting

$$
g=g_{1} \oplus g_{2},
$$

one obtains a mem-structure on $\xi$. So we have 
Proposition 2.2. A mem-structure $(J, g)$ on $\xi$ is equivalent to a pair of supplementary subbundles $\xi_{1}$ and $\xi_{2}$ respectively endowed with a para-Hermitian structure $\left(J_{1}, g_{1}\right)$ and an indefinite Hermitian structure $\left(J_{2}, g_{2}\right)$.

Remark 2.2. If $(J, g)$ is a mem-structure on $\xi$, then we have: $\operatorname{rank} \xi_{1}$ and $\operatorname{rank} \xi_{2}$ are even; trace $J_{1}=\operatorname{trace} J_{2}=0$; $\operatorname{sign} g_{1}=0$.

Setting for a mem-structure $(J, g)$ on $\xi$ :

$$
\Omega(X, Y)=g(J X, Y), \quad \Omega_{i}(X, Y)=g_{i}\left(J_{i} X, Y\right), \quad i=1,2,
$$

it follows that $\Omega, \Omega_{1}$, and $\Omega_{2}$ are 2-forms which determine almost symplectic structures of $\xi, \xi_{1}$ and $\xi_{2}$, so that

$$
\Omega=\Omega_{1} \oplus \Omega_{2} .
$$

These 2-forms satisfy

$$
\delta_{J} \Omega=0, \quad \delta_{J_{1}} \Omega_{1}=0, \quad \delta_{J_{2}} \Omega_{2}=0 .
$$

Remark 2.3. The meaning of conditions (2.2), (2.3) and (2.5) is the following: The groups of automorphisms of $\mathfrak{X}\left(\xi_{1}\right), \mathfrak{X}\left(\xi_{2}\right)$, and $\mathfrak{X}(\xi)$ given by

$$
\alpha_{t}=I_{1} \cosh t+J_{1} \sinh t, \quad \beta_{t}=I_{2} \cos t+J_{2} \sin t, \quad \gamma_{t}=\alpha_{t} \oplus \beta_{t},
$$

$t \in \mathbb{R}$, determine actions on the tensor algebras $\mathcal{T}\left(\xi_{1}\right), \mathcal{T}\left(\xi_{2}\right)$, and $\mathcal{T}(\xi)$, which respectively preserve the structures $\left(J_{1}, g_{1}, \Omega_{1}\right),\left(J_{2}, g_{2}, \Omega_{2}\right)$, and $(J, g, \Omega)$.

\section{Compatible connections}

\subsection{The general case}

Definition 3.1. A connection $D$ on the vector bundle $\xi$ is said to be compatible with an em-structure $J$ if

$$
D J=0 .
$$

From this it follows $D P=0$, hence $D$ preserves the subbundles $\xi_{1}$ and $\xi_{2}$, i.e., for $X \in \mathfrak{X}(M), Y_{1} \in \mathfrak{X}\left(\xi_{1}\right), Y_{2} \in \mathfrak{X}\left(\xi_{2}\right)$, one has $D_{X} Y_{1} \in \mathfrak{X}\left(\xi_{1}\right), D_{X} Y_{2} \in \mathfrak{X}\left(\xi_{2}\right)$. Setting then

$$
D_{X}^{1} Y_{1}=D_{X} Y_{1}, D_{X}^{2} Y_{2}=D_{X} Y_{2}, \quad X \in \mathfrak{X}(M), Y_{1} \in \mathfrak{X}\left(\xi_{1}\right), Y_{2} \in \mathfrak{X}\left(\xi_{2}\right),
$$

we have that $D^{1}$ and $D^{2}$ are respectively connections on $\xi_{1}$ and $\xi_{2}$, so that

$$
D_{X}=D_{X}^{1} \circ P_{1}+D_{X}^{2} \circ P_{2}, \quad D_{X}^{1} J_{1}=0, \quad D_{X}^{2} J_{2}=0, \quad X \in \mathfrak{X}(M) .
$$

Conversely, if $D^{1}$ and $D^{2}$ are respectively connections on $\xi_{1}$ and $\xi_{2}$, then $D$ given as in (3.2) is a connection on $\xi$ satisfying $D P=0$. If $D_{1}$ and $D_{2}$ satisfy the respective conditions in (3.2), then $D$ satisfies (3.1) too. Thus, it follows 
Proposition 3.1. A connection $D$ on $\xi$ is compatible with the em-structure $J$ if and only if there exist two connections $D^{1}$ on $\xi_{1}$ and $D^{2}$ on $\xi_{2}$, respectively compatible with the product structure $J_{1}$ and the complex structure $J_{2}$, so that

$$
D=D^{1} \circ P_{1}+D^{2} \circ P_{2} .
$$

Consider now on the subbundles $\xi_{i}$ of $\xi$, the operators $\Phi_{J_{i}}$ and $\Psi_{J_{i}}$ given by

$$
\left(\Phi_{J_{i}} D^{i}\right)_{X}=\frac{1}{2}\left(D_{X}^{i}+J_{i}^{-1} \circ D_{X}^{i} \circ J_{i}\right),\left(\Psi_{J_{i}} \mathcal{A}^{i}\right)_{X}=\frac{1}{2}\left(\mathcal{A}_{X}^{i}+J_{i}^{-1} \circ \mathcal{A}_{X}^{i} \circ J_{i}\right),
$$

where $X \in \mathfrak{X}(M), D^{i}$ is a connection on $\xi_{i}$, and $\mathcal{A}^{i} \in \Lambda^{1}(M) \otimes \mathfrak{X}\left(\xi_{i}\right) \otimes \Lambda^{1}\left(\xi_{i}\right)$ (now and in the sequel we take $i=1,2$ ). From $[1,13]$ and Proposition 3.1 we obtain

Proposition 3.2. The set of connections on $\xi$ compatible with the em-structure $J$ is given by

$$
D_{X}=\left\{\left(\Phi_{J_{1}} D^{\circ 1}\right)_{X}+\left(\Psi_{J_{1}} \mathcal{A}^{1}\right)_{X}\right\} \circ P_{1}+\left\{\left(\Phi_{J_{2}} D^{\circ 2}\right)_{X}+\left(\Psi_{J_{2}} \mathcal{A}^{2}\right)_{X}\right\} \circ P_{2},
$$

where $X \in \mathfrak{X}(M)$ and $D^{\circ i}$ is an arbitrary fixed connection on $\xi_{i}, \mathcal{A}^{i}$ denotes any element of $\Lambda^{1}(M) \otimes \mathfrak{X}\left(\xi_{i}\right) \otimes \Lambda^{1}\left(\xi_{i}\right)$, and $\Phi_{J_{i}}, \Psi_{J_{i}}$ are given by (3.4).

Definition 3.2. A connection $D$ on $\xi$ is said to be compatible with the memstructure $(J, g)$ if

$$
D J=0, \quad D g=0
$$

From which it follows: $D P=0 ; D=D^{1} \circ P_{1}+D^{2} \circ P_{2}$, where $D^{i}$ are the restrictions of $D$ to $\xi_{1}$ and $\xi_{2} ; D^{i} J_{i}=0$; and $D^{i} g_{i}=0$. Conversely, if $D^{1}$ and $D^{2}$ are connections on $\xi_{1}$ and $\xi_{2}$, compatible with the para-Hermitian structure $\left(J_{1}, g_{1}\right)$ and the indefinite Hermitian structure $\left(J_{2}, g_{2}\right)$ respectively, then the connection $D$ given by (3.3) is compatible with the mem-structure $(J, g)$ on $\xi$. So, we have

Proposition 3.3. A connection $D$ on $\xi$ is compatible with the mem-structure $(J, g)$ on $\xi$, if and only if there are two connections $D^{1}$ and $D^{2}$ on the subbundles $\xi_{1}$ and $\xi_{2}$, respectively compatible with the para-Hermitian structure $\left(J_{1}, g_{1}\right)$ and the indefinite Hermitian structure $\left(J_{2}, g_{2}\right)$, so that $D$ is given by (3.3).

Setting then

$$
\left(\Phi_{g_{i}} D^{i}\right)_{X}=\frac{1}{2}\left(D_{X}^{i}+g_{i}^{-1} \circ D_{X}^{i} \circ g_{i}\right),\left(\Psi_{g_{i}} \mathcal{A}^{i}\right)_{X}=\frac{1}{2}\left(\mathcal{A}_{X}^{i}+g_{i}^{-1} \circ \mathcal{A}_{X}^{i} \circ g_{i}\right),
$$

we obtain from [1], Prop. 3.3, and (2.4)

Proposition 3.4. The set of connections on $\xi$ compatible with the mem-structure $(J, g)$ is given by

$$
\begin{aligned}
D_{X}=\left\{\left(\left(\Phi_{g_{1}} \circ \Phi_{J_{1}}\right) D^{\circ 1}\right)_{X}\right. & \left.+\left(\left(\Psi_{g_{1}} \circ \Psi_{J_{1}}\right) \mathcal{A}^{1}\right)_{X}\right\} \circ P_{1} \\
+ & \left\{\left(\left(\Phi_{g_{2}} \circ \Phi_{J_{2}}\right) D^{\circ 2}\right)_{X}+\left(\left(\Psi_{g_{2}} \circ \Psi_{J_{2}}\right) \mathcal{A}^{2}\right)_{X}\right\} \circ P_{2},
\end{aligned}
$$

where $D^{\circ i}$ is an arbitrary fixed connection on $\xi_{i}, \mathcal{A}^{i} \in \Lambda^{1}(M) \otimes \mathfrak{X}\left(\xi_{i}\right) \otimes \Lambda^{1}\left(\xi_{i}\right)$, and $\Phi_{J_{i}}, \Phi_{g_{i}}, \Psi_{J_{i}}, \Psi_{g_{i}}$ are given by (3.4) and (3.5). 


\subsection{The case of the tangent bundle}

We now consider the case of $\xi$ being the tangent bundle of the manifold $M$, i.e., $\xi=(T M, \pi, M)$. In this case, for a mem-structure $(J, g)$ on $M$, the pair $(P, g)$ is a pseudo-Riemannian almost product structure on $M$, and $\left(J_{1}, g_{1}\right),\left(J_{2}, g_{2}\right)$, are respectively a para-Hermitian [4] and an indefinite Hermitian structure [10] on $\xi_{1}$ and $\xi_{2}$. If $\nabla$ is a linear connection on $M$, compatible with $P$, i.e., $\nabla P=0$, then its restrictions $\nabla^{1}$ and $\nabla^{2}$ to $\xi_{1}$ and $\xi_{2}$ are connections on these subbundles. If $T$ is the torsion tensor of $\nabla$, we shall call torsion tensor of $\nabla^{i}$ to the tensor fields $T^{i}$ given by $T^{i}=\left.P_{i} \circ T\right|_{\xi_{i}}$, or in more detail

$$
T^{i}\left(X_{i}, Y_{i}\right)=\nabla_{X_{i}} Y_{i}-\nabla_{Y_{i}} X_{i}-P_{i}\left[X_{i}, Y_{i}\right], \quad X_{i}, Y_{i} \in \mathfrak{X}\left(\xi_{i}\right) .
$$

We call tensors of nonholonomy of the distributions $\xi_{1}$ and $\xi_{2}$ to the tensor fields $S^{1}=\left.P_{2} \circ T\right|_{\xi_{1}}$ and $S^{2}=\left.P_{1} \circ T\right|_{\xi_{2}}$, respectively. We obtain

$$
S^{1}\left(X_{1}, Y_{1}\right)=-P_{2}\left[X_{1}, Y_{1}\right], \quad S^{2}\left(X_{2}, Y_{2}\right)=-P_{1}\left[X_{2}, Y_{2}\right] .
$$

It follows

Proposition 3.5. The distribution $\xi_{1}$ (resp. $\left.\xi_{2}\right)$ is involutive if and only if $S^{1}=0\left(\right.$ resp. $\left.S^{2}=0\right)$.

After some computations we obtain from $[3,10,14]$

Proposition 3.6. For a mem-structure $(J, g)$ on a manifold $M$, there exists a unique linear connection $\nabla$ with torsion tensor $T$, satisfying the conditions

$$
\begin{aligned}
& \nabla P=0, \quad T(P X, Y)=T(X, P Y), \\
& \nabla_{X_{i}}^{i} J_{i}=0, \quad \nabla_{X_{i}}^{i} g_{i}=0, \quad T^{i}\left(J_{i} X, I_{i} Y\right)=T^{i}\left(I_{i} X, J_{i} Y\right) .
\end{aligned}
$$

Definition 3.3. We shall call the canonical connection associated to the memstructure $(J, g)$ on the manifold $M$ to the connection given by the conditions (3.6) and (3.7).

Remark 3.1. Notice that this connection slightly differs from that given in Theorem 5.3 in [14].

For the canonical connection we obtain from (3.6):

$$
\nabla_{X_{2}}^{1} Y_{1}=P_{1}\left[X_{2}, Y_{1}\right], \quad \nabla_{X_{1}}^{2} Y_{2}=P_{2}\left[X_{1}, Y_{2}\right] .
$$

Denoting by $\xi_{1}^{1}, \xi_{1}^{2}$ the eigen-subbundles of $J_{1}$ corresponding to $\varepsilon=+1, \varepsilon=-1$, by $\pi_{1}^{1}, \pi_{1}^{2}$ the projection maps of $\xi_{1}$ on $\xi_{1}^{1}$, and $\xi_{1}^{2}$ and by $X_{1}^{i}, Y_{1}^{i}$ any elements of $\mathfrak{X}\left(\xi_{1}^{i}\right)$, we obtain from the first equation in (3.7)

$$
\begin{aligned}
& \nabla_{X_{1}^{2}}^{1} Y_{1}^{1}=\pi_{1}^{1} P_{1}\left[X_{1}^{2}, Y_{1}^{1}\right], \quad \nabla_{X_{1}^{1}}^{1} Y_{1}^{2}=\pi_{1}^{2} P_{1}\left[X_{1}^{1}, Y_{1}^{2}\right], \\
& g_{1}\left(\nabla_{X_{1}^{1}}^{1} Y_{1}^{1}, Z_{1}^{2}\right)=X_{1}^{1} g_{1}\left(Y_{1}^{1}, Z_{1}^{2}\right)-g_{1}\left(\left[X_{1}^{1}, Z_{1}^{2}\right], Y_{1}^{1}\right), \\
& g_{1}\left(\nabla_{X_{1}^{2}}^{1} Y_{1}^{2}, Z_{1}^{1}\right)=X_{1}^{2} g_{1}\left(Y_{1}^{2}, Z_{1}^{1}\right)-g_{1}\left(\left[X_{1}^{2}, Z_{1}^{1}\right], Y_{1}^{2}\right) .
\end{aligned}
$$


From the second equation in (3.7) above it results, exactly as in [14, Th. 5.1], the expression for $\nabla_{X_{2}}^{2} Y_{2}$.

For $J$ and $g$ we obtain

$$
\begin{aligned}
& \left(\nabla_{X_{1}} J\right) Y_{1}=0, \quad\left(\nabla_{X_{2}} J\right) Y_{2}=0, \quad\left(\nabla_{X_{1}} J\right) Y_{2}=\left(\nabla_{X_{1}}^{2} J_{2}\right) Y_{2}, \\
& \left(\nabla_{X_{2}} J\right) Y_{1}=\left(\nabla_{X_{1}}^{1} J_{1}\right) Y_{1}, \quad\left(\nabla_{X_{1}} g\right)\left(Y_{1}, Z_{1}\right)=0, \quad\left(\nabla_{X_{2}} g\right)\left(Y_{2}, Z_{2}\right)=0, \\
& \quad\left(\nabla_{X_{2}} g\right)\left(Y_{1}, Z_{1}\right)=\left(L_{X_{2}} g\right)\left(Y_{1}, Z_{1}\right), \quad\left(\nabla_{X_{1}} g\right)\left(Y_{2}, Z_{2}\right)=\left(L_{X_{1}} g\right)\left(Y_{2}, Z_{2}\right),
\end{aligned}
$$

where $L$ stands for the Lie derivative.

\section{Structures of electromagnetic type on the to- tal space of a vector bundle}

Let $\xi=(E, \pi, M)$ be a vector bundle and $\left(x^{j}\right),\left(y^{a}\right),\left(x^{j}, y^{a}\right)$, local coordinates in adapted charts on $M, \xi$, and $E$, respectively. We denote by $\left(\partial_{j}\right),\left(e_{a}\right),\left(\partial_{j}, \partial_{a}\right)$ the corresponding local bases, where $\partial_{j}=\partial / \partial x^{j}, \partial_{a}=\partial / \partial y^{a}, j=1,2, \ldots, m$, $a, b, c=1,2, \ldots, n$ (see [2]). Setting for each $z=(x, y) \in E, V_{z} E=\operatorname{Ker} \pi_{* z}$, we obtain the vertical distribution and so the vertical subbundle of $T E$, denoted by $V E$. Let $C^{\infty v}=\left\{f^{v}=f \circ \pi: f \in C^{\infty}(M)\right\}$ be the subring of $C^{\infty}(E)$ naturally isomorphic to $C^{\infty}(M)$. Setting for each $\mu \in \Lambda^{1}(\xi)$, locally given by $\mu(x)=\mu_{a}(z) e^{a}$,

$$
\gamma(\mu)(z)=\mu_{a}(x) y^{a},
$$

we obtain a class of functions on $E$ enjoying the property that every vector field $A \in \mathfrak{X}(E)$ is uniquely determined by its values on those functions. The mapping $\gamma$ may be extended to tensor fields $S \in \mathcal{T}_{1}^{1}(\xi)$ by

$$
(\gamma S)(\gamma(\mu))=\gamma(\mu \circ S), \quad \mu \in \Lambda^{1}(\xi) .
$$

If $S(x)=S_{b}^{a}(x) e_{a} \otimes e^{b}$, then $\gamma S(z)=S_{b}^{a}(x) y^{b} \partial_{a}$, i.e., $\gamma S$ is a vertical vector field on $E$. Now, let $D$ be a connection on $\xi$ and $X \in \mathfrak{X}(M), u \in \mathfrak{X}(\xi)$. Setting

$$
X^{h}(\gamma \mu)=\gamma\left(D_{X} \mu\right), \quad u^{v}(\gamma \mu)=\mu(u) \circ \pi, \quad \mu \in \Lambda^{1}(\xi),
$$

we obtain two vector fields $X^{h}$ and $u^{v}$ on $E$, respectively called the horizontal lift of $X$ and the vertical lift of $u$. We have the useful formulas [2]:

$$
\begin{array}{r}
(f X)^{h}=f^{v} X^{h},(f u)^{v}=f^{v} u^{v},\left[X^{h}, Y^{h}\right]=[X, Y]^{h}-\gamma R_{X Y}^{D}, \quad\left[u^{v}, w^{v}\right]=0, \\
{\left[X^{h}, u^{v}\right]=\left(D_{X} u\right)^{v}, \quad f \in \mathbb{C}^{\infty}(M), X, Y \in \mathfrak{X}(M), u, w \in \mathfrak{X}(\xi) .}
\end{array}
$$

Now, putting

$$
Q\left(X^{h}\right)=X^{h}, \quad Q\left(u^{v}\right)=-X^{v}, \quad X \in \mathfrak{X}(M), u \in \mathfrak{X}(\xi),
$$

we obtain an almost product $Q$ structure on $E$ whose +1 and -1 eigendistributions, are respectively called the horizontal distribution $H E$ of the connection $D$ and the vertical distribution $V E$ of the bundle. 
For $f \in \mathcal{T}_{1}^{1}(M), \varphi \in \mathcal{T}_{1}^{1}(\xi), g \in \mathcal{T}_{2}(M), \psi \in \mathcal{T}_{2}(\xi)$, we define the horizontal lift or the vertical lift $f^{h}, \varphi^{v}, g^{h}, \psi^{v}$, respectively by

$$
\begin{array}{r}
f^{h}\left(X^{h}\right)=f(X)^{h}, \quad f^{h}\left(u^{v}\right)=0, \quad \varphi^{v}\left(X^{h}\right)=0, \quad \varphi^{v}\left(u^{v}\right)=\varphi(u)^{v}, \\
g^{h}\left(X^{h}, Y^{h}\right)=g(X, Y)^{v}, \quad g^{h}\left(X^{h}, u^{v}\right)=g^{h}\left(u^{v}, X^{h}\right)=g^{h}\left(u^{v}, w^{v}\right)=0, \\
\psi^{v}\left(X^{h}, Y^{h}\right)=\psi^{v}\left(X^{h}, u^{v}\right)=\psi^{v}\left(u^{v}, Y^{h}\right)=0, \quad \psi^{v}\left(u^{v}, w^{v}\right)=\psi(u, w)^{v}, \\
X, Y \in \mathfrak{X}(M), u, w \in \mathfrak{X}(\xi) .
\end{array}
$$

We then define the diagonal lifts $J$ and $G$ for the pairs $(f, \varphi)$ and $(g, \psi)$ by

$$
J=f^{h}+\varphi^{v}, \quad G=g^{h}+\psi^{v} .
$$

From (4.1) and (4.2) we have

$$
J^{n}\left(X^{h}\right)=\left(f^{n}(X)\right)^{h}, \quad J^{n}\left(u^{v}\right)=\left(\varphi^{n}(u)\right)^{v}, \quad n \in \mathbb{N}^{*} .
$$

So $J^{4}=I$, that is $J$ is an em-structure on $E$, if and only if $f^{4}=I_{1}$ and $\varphi^{4}=I_{2}$, that is, either $f$ and $\varphi$ are both em-structures or one is an em-structure and the other an almost product or almost complex structure, or finally $f$ is an almost product (resp. almost complex) and $\varphi$ is a complex (resp. product) structure on $M$ and $\xi$ respectively. In the sequel we only consider the last case.

Hence, let $J$ be an em-structure on the total space $E$ of $\xi$ given by the diagonal lift in the first equation in (4.2) of an almost product (resp. almost complex) structure $f$ on the base manifold $M$ and a complex (resp. product) structure $\varphi$ on the bundle $\xi$, that is, which satisfy

$$
f^{2}=\varepsilon I_{1}, \quad \varphi^{2}=-\varepsilon I_{2}, \quad \varepsilon=1(\text { resp. } \quad \varepsilon=-1),
$$

with respect to a connection $D$ on $\xi$. For the almost product structure $P$ associated to $J$, we obtain $P=\varepsilon Q$, that is, $P$ coincides up to the sign with the almost product structure $Q$ above associated to $D$.

Now, let $G$ be the diagonal lift in the second equation in (4.2), with respect to $D$, for the pair $(g, \psi)$ of metrics on $M$ and $\xi$. From (4.2) we obtain

$$
\delta_{J} G=\left(\delta_{f} g\right)^{h}+\left(\delta_{\varphi} \psi\right)^{v},
$$

and so $\delta_{J} G=0$ if and only if $\delta_{f} g=0$ and $\delta_{\varphi} \psi=0$. It follows

Proposition 4.1. The pair $(J, G)$ of diagonal lifts, with respect to a connection $D$ on $\xi$, of an almost product (resp. almost complex) structure $f$ on $M$ and a complex (resp. product) structure $\varphi$ of $\xi$, and the nondegenerate metrics $g$ on $M$ and $\psi$ on $\xi$, is a mem-structure on the total space $E$ of $\xi$ if and only if the pair $(f, g)$ is an almost para-Hermitian (resp. indefinite almost Hermitian) structure on $M$. The pair $(\varphi, \psi)$ is an indefinite Hermitian (resp. para-Hermitian) structure on $\xi$. 
Denoting by $\omega$ and $\tau$ the 2 -forms associated to the structures $(f, g)$ on $M$ and $(\varphi, \psi)$ on $\xi$, and by $\Omega_{1}, \Omega_{2}, \Omega$, the 2 -forms associated to the structures $\left(f^{h}, g^{h}\right)$ on $H E,\left(\varphi^{v}, \psi^{v}\right)$ on $V E$ and $(J, G)$ on $T E$, we obtain

$$
\Omega_{1}=\omega^{h}, \quad \Omega_{2}=\tau^{v}, \quad \Omega=\omega^{h} \oplus \tau^{v} .
$$

From the hypotheses of Prop. 4.1 it follows

$$
\delta_{f} g=0, \quad \delta_{f} \omega=0, \quad \delta_{\varphi} \psi=0, \quad \delta_{\varphi} \tau=0, \quad \delta_{J} G=0, \quad \delta_{J} \Omega=0 .
$$

Remark 4.1. The groups of automorphisms of $\mathfrak{X}(M), \mathfrak{X}(\xi), \mathfrak{X}(E)$, given respectively for $\varepsilon=1$ and $\varepsilon=-1$, by

$$
\begin{aligned}
& \alpha_{t}=I_{1} \cosh t+f \sinh t, \quad \beta_{t}=I_{2} \cos t+\varphi \sin t, \quad \gamma_{t}=\alpha_{t}^{h} \oplus \beta_{t}^{h}, \quad t \in \mathbb{R}, \\
& \alpha_{t}=I_{1} \cos t+f \sin t, \quad \beta_{t}=I_{2} \cosh t+\varphi \sinh t, \quad \gamma_{t}=\alpha_{t}^{h} \oplus \beta_{t}^{h}, \quad t \in \mathbb{R},
\end{aligned}
$$

determine on the tensor algebras $\mathcal{T}(M), \mathcal{T}(\xi)$, and $\mathcal{T}(E)$, actions which preserve the structures $(f, g, \omega),(\varphi, \psi, \tau)$ and $(J, G, \Omega)$.

For two connections $\nabla$ on $M$ and $D$ on $\xi$, we define the horizontal lift $\nabla^{h}$ on the subbundle $H E$ and the vertical lift $D^{v}$ on the subbundle $V E$ (each one with respect to the connection $D$ ), respectively by

$$
\nabla_{X^{h}}^{h} Y^{h}=\left(\nabla_{X} Y\right)^{h}, \quad \nabla_{u^{v}}^{h} Y^{h}=0, \quad D_{X^{h}}^{v} w^{v}=\left(D_{X} w\right)^{v}, \quad D_{u^{v}}^{v} w^{v}=0 .
$$

Putting them

$$
\mathcal{D}_{A} X=\nabla_{A}^{h} H X+D_{A}^{v} V X, \quad A, X \in \mathfrak{X}(E),
$$

where $H$ and $V$ denote the horizontal and vertical projectors of $T E$ on $H E$ and $V E$, we obtain a linear connection $\mathcal{D}$ on $E$, called the diagonal lift of the pair $(\nabla, D)$ with respect to the connection $D$ (see [2]), whose restrictions to the subbundles $\xi_{1}=H E$ and $\xi_{2}=V E$ are $\mathcal{D}_{1}=\nabla^{h}$ and $\mathcal{D}_{2}=D^{v}$. The nonvanishing components of the torsion and curvature tensors of $\mathcal{D}$ are given by

$$
\begin{aligned}
& \mathcal{T}\left(X^{h}, Y^{h}\right)=T^{\nabla}(X, Y)^{h}+\gamma R_{X Y}^{D}, \\
& \mathcal{R}_{X^{h} Y^{h}} Z^{h}=\left(R_{X Y}^{\nabla} Z\right)^{h}, \quad \mathcal{R}_{X^{h} Y^{h}} u^{v}=\left(R_{X Y}^{D} u\right)^{v},
\end{aligned}
$$

where $T^{\nabla}, R^{\nabla}$, and $R^{D}$ stand for the torsion tensor of $\nabla$ and the curvature tensors of $\nabla$ and $D$.

For the covariant derivatives, with respect to $\mathcal{D}$, of the horizontal lift of $f$ and $g$, and the vertical lift of $\varphi$ and $\psi$ we obtain

$$
\begin{array}{cccc}
\mathcal{D}_{X^{h}} f^{h}=\left(\nabla_{X} f\right)^{h}, & \mathcal{D}_{u^{v}} f^{h}=0, & \mathcal{D}_{X^{h}} g^{h}=\left(\nabla_{X} g\right)^{h}, & \mathcal{D}_{u^{v}} g^{h}=0, \\
\mathcal{D}_{X^{h}} \varphi^{v}=\left(D_{X} \varphi\right)^{v}, & \mathcal{D}_{u^{v}} \varphi^{v}=0, & \mathcal{D}_{X^{h}} \psi^{v}=\left(D_{X} \psi\right)^{v}, & \mathcal{D}_{u^{v}} \psi^{v}=0 .
\end{array}
$$


So, for the diagonal lifts $J$ and $G$ of the pairs $(f, \varphi)$ and $(g, \psi)$, it follows

$$
\begin{array}{ll}
\mathcal{D}_{X^{h}} J=\left(\nabla_{X} f\right)^{h}+\left(D_{X} \varphi\right)^{v}, & \mathcal{D}_{u^{v}} J=0, \\
\mathcal{D}_{X^{h}} G=\left(\nabla_{X} g\right)^{h}+\left(D_{X} \psi\right)^{v}, & \mathcal{D}_{u^{v}} G=0 .
\end{array}
$$

Hence, $\mathcal{D} J=0$ if and only if $\nabla f=0, D \varphi=0$; and $\mathcal{D} G=0$ if and only if $\nabla g=0, D \psi=0$. From (4.3) and (4.4) it follows, for $P=J^{2}$, that $\mathcal{D} P=0$ and $\mathcal{T} \circ P \times I=\mathcal{T} \circ I \times P$ for any connections $\nabla$ on $M$ and $D$ on $\xi$. After that we have

$$
\begin{gathered}
\nabla_{X^{h}}^{h} g^{h}=\left(\nabla_{X} g\right)^{h}, \quad D_{u^{v}}^{v} \varphi^{v}=0, \quad D_{u^{v}}^{v} \psi^{v}=0, \\
\nabla_{X^{h}}^{h} f^{h}=\left(\nabla_{X} f\right)^{h}, \quad \mathcal{T}^{1}\left(f^{h} X, I_{1} Y\right)=\left(T^{\nabla}\left(f X, I_{1} Y\right)\right)^{h}, \quad \mathcal{T}^{2}\left(\varphi^{v} X, I_{2} Y\right)=0,
\end{gathered}
$$

where $\mathcal{T}^{1}=\left.H \circ \mathcal{T}\right|_{H E}$ and $\mathcal{T}^{2}=\left.V \circ \mathcal{T}\right|_{V E}$. So we obtain

Proposition 4.2. The diagonal lift $\mathcal{D}$ on $E$, for the connections $\nabla$ on $M$ and $D$ on $\xi$, is the canonical connection associated to the mem-structure $(J, G)$ if and only if

$$
\nabla f=0, \quad \nabla g=0, \quad T^{\nabla}(f X, Y)=T^{\nabla}(X, f Y),
$$

i.e., the connection $\nabla$ is the canonical connection $[2,10]$ associated to the almost para-Hermitian (resp. indefinite almost Hermitian) structure $(f, g)$ on $M$.

Also from (4.3) and (4.4) we obtain $\mathcal{D} G=0$ and $\mathcal{T}=0$ if and only if $\nabla g=0$, $T^{\nabla}=0, R^{D}=0$ and $D \psi=0$. Hence we have

Proposition 4.3. The diagonal lift $\mathcal{D}$ of the pair of connections $(\nabla, D)$ coincides with the Levi-Civita connection of $G$ if and only if $\nabla$ is the Levi-Civita connection of $g, D$ has vanishing curvature and $\psi$ is covariant constant.

For the Nijenhuis tensor of $J$,

$$
N_{J}(A, B)=[J A, J B]+J^{2}[A, B]-J[J A, B]-J[A, J B], \quad A, B \in \mathfrak{X}(E),
$$

we obtain

$$
\begin{aligned}
& \text { (4.5) } N_{J}\left(X^{h}, Y^{h}\right)=N_{f}(X, Y)^{h}+\gamma\left(\varepsilon R_{X Y}^{D}-R_{f X f Y}^{D}+\varphi \circ\left(R_{f X Y}^{D}+R_{X f Y}^{D}\right)\right), \\
& N_{J}\left(X^{h}, u^{v}\right)=\left(D_{f X} \varphi u-\varepsilon D_{X} u-\varphi \circ\left(D_{f X} u+D_{X} \varphi u\right)\right)^{v}, \quad N_{J}\left(u^{v}, w^{v}\right)=0 .
\end{aligned}
$$

It follows

Proposition 4.4. The mem-structure $J$ is integrable (i.e., $N_{J}=0$, see [8]) if and only if $f$ is a product (resp. a complex) structure in $M$, the connection $D$ has vanishing curvature and the complex (resp. product) structure $\varphi$ on $\xi$ is covariant constant.

For the exterior differential of the 2 -form $\Omega$ associated to the mem-structure $(J, G)$ we obtain

$$
\begin{gathered}
d \Omega\left(X^{h}, Y^{h}, Z^{h}\right)=d \omega(X, Y, Z)^{v}, \quad 3 d \Omega\left(X^{h}, Y^{h}, w^{v}\right)=-\gamma\left(i_{w} \tau \circ R_{X Y}^{D}\right), \\
3 d \Omega\left(X^{h}, u^{v}, w^{v}\right)=D_{X} \tau(u, w)^{v}, \quad d \Omega\left(u^{v}, v^{v}, w^{v}\right)=0 .
\end{gathered}
$$

Hence 
Proposition 4.5. The almost symplectic structure $\Omega$ associated to the memstructure $(J, G)$ on $E$ is integrable (i.e., $d \Omega=0)$ if and only if the structure $(f, g)$ is almost para-Kähler (resp. indefinite almost Kähler), the connection D has vanishing curvature, and the 2 -form $\tau$ on $\xi$ is covariant constant.

Finally we obtain

Proposition 4.6. For the mem-structure $(J, G)$ on $E$, the structures $J$ and $\Omega$ are simultaneously integrable if and only if the structure $(f, g)$ is a para-Kähler (resp. indefinite Kähler) structure on $M, D$ has vanishing curvature and the pair $(\varphi, \psi)$ is covariant constant.

\section{References}

[1] V. Cruceanu, Connexions compatibles avec certaines structures sur un fibré vectoriel banachique, Czechoslovak Math. J. 24 (1974) 126-142.

[2] V. Cruceanu, A new definition for certain lifts on a vector bundle, An. Ştiinţ. Univ. "Al. I. Cuza" Iaşi 42 (1996) 59-73.

[3] V. Cruceanu \& F. Etayo, On almost para-Hermitian manifolds, Algebras Groups Geom. (to appear in 1999).

[4] V. Cruceanu, P. Fortuny \& P.M. Gadea, A survey on Paracomplex Geometry, Rocky Mountain J. Math. 26 (1996) 83-115.

[5] F. Etayo \& E. Reyes, Normality and structure transfer in $\left(J^{4}=1\right)$-manifolds, Rend. Sem. Fac. Sci. Univ. Cagliari 62 (1992) 1-7.

[6] J.M. Hernando \& P.M. Gadea, Sobre ciertas estructuras polinómicas, Act. VII Jornadas Hisp.-Lusit., S. Feliu de Guixols, vol. 1, 173-176 (1980).

[7] J.M. Hernando, P.M. Gadea \& A. Montesinos Amilibia, G-structures defined by a tensor field of electromagnetic type, Rend. Circ. Mat. Palermo (2) 34 (1985) $202-218$.

[8] J.M. Hernando, E. Reyes \& P.M. Gadea, Integrability of tensor structures of electromagnetic type, Publ. Inst. Math. (Beograd) (N.S.) 37 (1985) 113-122.

[9] V. Hlavatý, Geometry of Einstein's unified field theory, P. Noordhoff, 1958.

[10] S. Kobayashi \& K. Nomizu, Foundations of Differential Geometry, Intersc. Publ., 1963 and 1969.

[11] R.S. Mishra, Structures in electromagnetic tensor fields, Tensor (N.S.) 30 (1976) $145-156$.

[12] R. Miron \& M. Anastasiei, Vector bundles and Lagrange spaces with applications in Relativity, Balkan Soc. Geom. Monographs and Textbooks, n. 1, 1998.

[13] E. Reyes, A. Montesinos Amilibia \& P.M. Gadea, Connections making parallel a metric $\left(J^{4}=1\right)$-structure, An. Ştiinţ. Univ. "Al. I. Cuza" Iaşi 28 (1982) 49-54.

[14] E. Reyes, A. Montesinos Amilibia \& P.M. Gadea, Connections partially adapted to a metric $\left(J^{4}=1\right)$-structure, Colloq. Math. 54 (1987) 216-229. 


\section{Authors' addresses:}

Encarna Reyes Iglesias: Department of Mathematics, E.T.S. of Architecture, University of Valladolid, Av. de Salamanca s/n, 47014-Valladolid, Spain. ereyes@cpd.uva.es Vasile Cruceanu: Department of Mathematics, University "Al. I. Cuza", 6600-Iaşi, Romania.cruv@uaic.ro

Pedro Martínez Gadea: Institute of Mathematics and Fundamental Physics, CSIC, Serrano 123, 28006-Madrid, Spain. pmgadea@iec.csic.es 